Pacific Journal of Mathematics

DEGENERATE SECANT VARIETIES AND A PROBLEM ON 


\section{DEGENERATE SECANT VARIETIES AND A PROBLEM ON MATRICES}

\section{NORMAN GOLDSTEIN}

We show that if a developable ruled surface of a curve in complex projective space has a degenerate secant variety, then the surface already lies in a $\mathbb{P}^{4}$. This result eliminates a redundancy in the list of Griffiths and Harris, of surfaces that have degenerate secant varieties.

1. Introduction. A $d$-dimensional variety $X \subset \mathbb{P}_{\mathbb{C}}^{N}, N \geq 2 d+1$, is said to have a degenerate secant variety, $\operatorname{Sec}(X)$, when $\operatorname{dim}(\operatorname{Sec}(X)) \leq 2 d$. In [1, Results 5.37, 6.16-18], Griffiths and Harris prove the Proposition: Let $X \subset \mathbb{P}^{N}$ be a surface having a degenerate secant variety. Then either (i) $X \subset \mathbb{P}^{4}$, (ii) $X$ is a cone, (iii) $X$ is the Veronese surface, or (iv) $X$ is developable.

It is easy to show that any of conditions (i), (ii) or (iii) implies that $X$ has a degenerate secant variety. The main contribution of the present paper is the Proposition (3.5): If a developable surface, $X$, has a degenerate secant variety, then $X$ is contained in a $\mathbb{P}^{4}$.

Combining the above two results, we obtain

TheOREM (1.0). A surface $X \subset \mathbb{P}^{N}$ has a degenerate secant variety precisely when one of conditions (i), (ii) or (iii) above is satisfied.

It is interesting to note that a developable surface always has a degenerate tangent variety i.e.

$$
\operatorname{dim}(\operatorname{Tan}(X)) \leq 3 \quad[1, \text { Result 5.37]; }
$$

this also follows from our Lemma (3.3).

We will see in $\S 3$ that a variety $X^{d} \subset \mathbb{P}^{N}$ gives rise to a family of $(N-d) \times(d+1)$ matrices $\{A(x)\}$ where $x$ belongs to some polydisc $U \subset \mathbb{C}^{d}$. Let | | denote "determinant". Result (3.2.1) states that

$$
\begin{aligned}
& \operatorname{dim} \operatorname{Sec}(X) \leq 2 d \\
& \Leftrightarrow \operatorname{rank}(A(x)-A(y)) \leq d \quad \forall x, y \in U \\
& \Leftrightarrow\left|A^{I}(x)-A^{I}(y)\right|=0 \quad \text { for all }(d+1) \text {-tuples of rows } \\
& \\
& \quad I=\left(i_{0}, i_{1}, \ldots, i_{d}\right) .
\end{aligned}
$$


The matrix problem inspired by this condition of degeneracy is:

Characterize those 1-parameter analytic families of $n \times n$ matrices $\{A(t)\}$ ( $t$ belongs to a disc $U \subset \mathbb{C}$ ) such that

$$
|A(t)-A(s)|=0 \quad \forall s, t \in U .
$$

We say that such families are degenerate. For $n=1$, of course, "degenerate" is the same as "constant". Letting $s \rightarrow t$ in (1.1), we find that $\left|A^{\prime}(t)\right|=0$ for a degenerate family $\{A(t)\}$; however it is easy to construct examples where this condition alone does not imply degeneracy of the family. The next result includes the solution for $n=2$ of the degeneracy problem (1.1).

Proposition (4.5). Let $\{A(t)\}$ be an $n \times n$ family such that $A^{\prime}(t)$ has rank 1 for all $t \in U$. Then $\{A(t)\}$ is degenerate precisely when

$$
\text { there are constant vectors } u, w(u \neq 0) \text { such that either }
$$

$$
A(t) u=w \quad \text { or } \quad u^{t} A(t)=w^{t} \quad \forall t \in U .
$$

Of course, for any $n \times n$ family, if condition (1.2) is satisfied then the family is degenerate. We call such families trivially degenerate. When the family arises geometrically, as described in $\S 3$, then the two cases (1.2) of trivial degeneracy correspond, respectively, to $X$ being a cone or $X$ being contained in a hyperplane.

For $n \geq 3$, there are nontrivially degenerate families. The Veronese surface furnishes such an example:

$$
A(x, y)=\left(\begin{array}{ccc}
x^{2} & 2 x & 0 \\
y^{2} & 0 & 2 y \\
x y & y & x
\end{array}\right)
$$

Here, $x$ and $y$ may be viewed as functions of $t$.

I do not know the structure of the general degenerate family for $n \geq 3$. Moreover, surfaces give rise to $3 \times 3$ families. However, when the surface, $X$, is developable, the family is actually of 1 parameter and $A^{\prime}(t)$ has rank 1. We then apply Proposition (4.5) and deduce that $X$ lies in a hyperplane when $\operatorname{Sec}(X)$ is degenerate.

Motivation to study secant varieties of surfaces arose from the Definition ([2, p. 257]): Let $\mathscr{Q} \subset \mathbb{P}^{5}$ be a smooth quadric hypersurface. A surface $X \subset \mathscr{Q}$ is said to have rank $r$ when for generic $z \in \mathscr{Q}$ there are precisely $r$ sets $\left\{x_{1}, x_{2}\right\} \subset X, x_{1} \neq x_{2}$, such that the line through $x_{1}$ and $x_{2}$ passes through $z$. 
One can show that $\operatorname{rank}(X)=0$ precisely when $\operatorname{Sec}(X) \neq \mathbb{P}^{5}$; this happens exactly when $X \subset \mathbb{P}^{4}$ or $X$ is the Veronese surface. (A cone in $\mathscr{Q}$ is contained in the $\mathbb{P}^{4}$ tangent to $\mathscr{Q}$ at the vertex of the cone.)

2. Notation. The setting for htis article is $N$-dimensional complex projective space, $\mathbb{P}^{4}$; it is the set of all linear 1-dimensional subspaces of $\mathbb{C}^{N+1}$. The methods we use are local and analytic in nature. For simplicity of presentation, however, we will consider only algebraic subvarieties of $\mathbb{P}^{N}$, i.e. Zariski open subsets of closed subvarieties of $\mathbb{P}^{N}$.

A line in $\mathbb{P}^{N}$ is a linear $\mathbb{P}^{1} \subset \mathbb{P}^{N}$. A line is said to be a secant line to a variety $X \subset \mathbb{P}^{N}$ when the line contains at least two points of $X$. The secant variety of $X, \operatorname{Sec}(X)$, is the closure of the union of all the secant lines to $X$.

Homogeneous coordinates in $\mathbb{P}^{N}$ are denoted by $\left(z_{0}, \ldots, z_{N}\right)$. Let $X$ be a $d$-dimensional subvariety of $\mathbb{P}^{N}$. The regular (i.e. manifold) points of $X$ are denoted by $X_{\text {reg }}$. Let $\Delta \subset X \times X$ be the diagonal, and $X_{0} \subset X_{\text {reg }}$ the open subset lying in $\left\{z_{0} \neq 0\right\}$. Let $\mathscr{X}_{0}=\left(X_{0} \times X_{0} \backslash \Delta\right) \times \mathbb{P}^{1}$, and $\sigma$ : $\mathscr{X}_{0} \rightarrow \mathbb{P}^{N}$ the map defined by $\sigma(x, y, \lambda, \mu)=\lambda x+\mu y$, where $x_{0}=y_{0}=1$ and $(\lambda, \mu) \in \mathbb{P}^{1}$. Then $\mathscr{X}_{0}$ is a $2 d+1$ dimensional smooth variety and $\sigma\left(\mathscr{X}_{0}\right)$ is a dense constructible subset of $\operatorname{Sec}(X)$. Assuming that $N \geq 2 d+$ 1 , we say that $\operatorname{Sec}(X)$ is degenerate when $\operatorname{dim} \operatorname{Sec}(X) \leq 2 d$. Sard's theorem provides a local criterion for degeneracy.

$\operatorname{Sec}(X)$ is degenerate $\Leftrightarrow$ the differential rank of $\sigma$ is at most $2 d$ at each point of $\mathscr{X}_{0}$.

Unless otherwise stated, a $\mathbb{P}^{r} \subset \mathbb{P}^{N}$ refers to a linear subspace. When $x \in X_{\text {reg }}$, there is a well-defined $\mathbb{P}^{d}$ tangent to $X$ at $x$, and we denote it as $\mathbb{P}_{x} X$.

A variety $X \subset \mathbb{P}^{N}$ is called a cone when there is a subvariety $Y \subset \mathbb{P}^{N}$ and a point $z \in \mathbb{P}^{N}$ such that $X$ is the union of all the lines that contain $z$ and meet $Y$.

We are mainly concerned with surfaces, i.e. 2-dimensional varieties. The Veronese surface is a smooth surface in $\mathbb{P}^{5}$ of degree 4 , and isomorphic to $\mathbb{P}^{2}$. Up to a regular isomorphism of $\mathbb{P}^{5}$, it is the image of the map $\mathbb{P}^{2} \rightarrow \mathbb{P}^{5}$ given by $(x, y, z) \mapsto\left(x^{2}, y^{2}, z^{2}, x y, x z, y z\right)$. A surface is called developable if there is a curve $C \subset \mathbb{P}^{N}$ such that $X$ is (the closure of) the union of all the tangent lines to $C$.

\section{Degeneracy and matrices.}

(3.0) Definition. A family of $m \times n$ matrices $\{A(x)\}, x \in U$, is said to be a degenerate family when $\operatorname{rank}(A(x)-A(y))<\min (m, n)$ for all $x, y$ belonging to $U$. 
In this section we establish the connection between degenerate secant varieties and degenerate families of matrices. We prove the main result (3.5), but relegate the matrix calculations to the final section of the paper.

The differential criterion (2.0) for degeneracy translates into
$\operatorname{Sec}(X)$ is degenerate $\Leftrightarrow \mathbb{P}_{x} X \cap \mathbb{P}_{y} X \neq \varnothing$
$\forall x, y \in X_{\text {reg }}$.

A proof of this statement is also in $[1, \S 6.2]$.

Without loss of generality, let $U \subset \mathbb{C}^{d}$ be an open set, and $U \rightarrow X$ a parametrization of (a Euclidean open subset of) $X$, given by $x \mapsto$ $(1, x, f(x))^{t}$, where $f: U \rightarrow \mathbb{C}^{N-d}$. In terms of these coordinates, $\mathbb{P}_{x} X$ is the linear space spanned by the columns of the $(N+1) \times(d+1)$ matrix

$$
\left(\begin{array}{cccc}
1 & 0 & \cdots & 0 \\
x & & I_{d} & \\
f(x) & & \partial f / \partial x &
\end{array}\right)
$$

where $I_{d}$ is the identity $d \times d$ matrix and $\partial f / \partial x$ is the Jacobian matrix of $f$. Let $A(x)$ be the $(N-d) \times(d+1)$ matrix

$$
\left(f(x)-\frac{\partial f}{\partial x} x \vdots \frac{\partial f}{\partial x}\right)
$$

so that $\mathbb{P}_{x} X$ is spanned by the columns of

$$
\left(\begin{array}{c}
I_{d+1} \\
A(x)
\end{array}\right)
$$

By (3.1) and (3.2),

(3.2.1) $\operatorname{Sec}(X)$ is degenerate $\Leftrightarrow \operatorname{rk}(A(x)-A(y)) \leq d \quad \forall x, y \in U$,

i.e. $\operatorname{Sec}(X)$ is degenerate precisely when $\{A(x)\}, x \in U$, form a degenerate family.

For the rest of this section, let $X$ be the developable surface of a curve $C \subset \mathbb{P}^{N}$.

Lemma (3.3). For generic $x \in X$, we have that $\mathbb{P}_{x} X=\mathbb{P}_{t}^{(2)} C$, the $2^{\text {nd }}$ osculating space to the curve $C$ at the point $t$ where the line containing $x$ is tangent to $C$.

Proof. Let $t \mapsto(1, t, g(t))^{t}$ parametrize a Euclidean neighborhood in C. So,

$$
(s, t) \mapsto(1, t, g)^{t}+s\left(0,1, g^{\prime}\right)^{t}=\left(1, s+t, g+s g^{\prime}\right)^{t}
$$


contains a parametrization of an open subset of $X$, and $\mathbb{P}_{x} X$ is spanned by the columns of

$$
\left(\begin{array}{ccc}
1 & 0 & 0 \\
t+s & 1 & 1 \\
g+s g^{\prime} & g^{\prime} & g^{\prime}+s g^{\prime \prime}
\end{array}\right)
$$

i.e. $\mathbb{P}_{x} X$ is spanned by the columns of

$$
\left(\begin{array}{ccc}
1 & 0 & 0 \\
t & 1 & 0 \\
g & g^{\prime} & g^{\prime \prime}
\end{array}\right)
$$

which also span $\mathbb{P}_{t}^{(2)} C$.

Assume, now, that $\operatorname{Sec}(X)$ is degenerate. We next show

Proposition (3.5). The developable surface, $X$, with a degenerate secant variety, is contained in a $\mathbb{P}^{4}$.

To show that $X \subset \mathbb{P}^{4}$, we may as well assume that $X$ is nondegenerate in $\mathbb{P}^{N}$ i.e. $X \not \subset \mathbb{P}^{N-1}$, and that $N \geq 5$. In fact, we may take $N=5$, since if $\pi$ is a projection into $\mathbb{P}^{5}$, then $\pi(X)$ is the developable surface of the curve $\pi(C), \operatorname{Sec}(\pi(X))=\pi(\operatorname{Sec}(X))$ is at most 4-dimensional, and if $\pi(X) \subset \mathbb{P}^{4}$ then $X \subset \mathbb{P}^{N-1}$.

We now construct the degenerate $3 \times 3$ family associated to $X$. By Lemma (3.3), this is a 1-parameter family; we use the notation of that lemma and write $g(t)=(a(t), B(t))^{t}$ where $a(t) \in \mathbb{C}$ and $B(t) \in \mathbb{C}^{3}$. We note that if $a^{\prime \prime} \equiv 0$ then $a=\lambda t+\mu$ and this implies that $C$, and hence $X$, is contained in a $\mathbb{P}^{4}$, viz. $z_{2}-\lambda z_{1}-\mu z_{0}=0$. So, we may assume that $a^{\prime \prime}(t) \neq 0$. With this in mind, we define the $3 \times 3$ matrix

$$
A(t)=\left(B-t B^{\prime}-\frac{a-t a^{\prime}}{a^{\prime \prime}} B^{\prime \prime}, B^{\prime}-\frac{a^{\prime}}{a^{\prime \prime}} B^{\prime \prime},\left(a^{\prime \prime}\right)^{-1} B^{\prime \prime}\right) .
$$

By performing column operations on the matrix (3.4), we find that the columns of

$$
\left(\begin{array}{c}
I_{3} \\
A(t)
\end{array}\right)
$$

span $\mathbb{P}_{t}^{(2)} C$, so that $\{A(t)\}, t \in U$, is the degenerate family associated to $X$. We differentiate and obtain that

$$
A^{\prime}(t)=(q E p E E)=E P^{t}
$$


has rank 1 , where

$$
\begin{array}{ll}
E=\left(B^{\prime \prime}\left(a^{\prime \prime}\right)^{-1}\right)^{\prime}, & p=t a^{\prime}-a, \\
P^{t}=(q p 1), & q=-a^{\prime}
\end{array}
$$

For a family $\{w(t)\}$ of 3-vectors, we denote the Wronskian $3 \times 3$ matrix by $\omega(w)=\left(w w^{\prime} w^{\prime \prime}\right)$. Using (3.6) one verifies that $|\omega(P)|=q^{\prime} p^{\prime \prime}$ $-q^{\prime \prime} p^{\prime}=-\left(a^{\prime \prime}\right)^{2} \neq 0$, so by Proposition (4.5) of the next section there is a constant 3-vector $u$ such that $u^{t} A^{\prime}=0$, i.e. $u^{t} E=0$. Thus

$$
\left(u^{t} B^{\prime \prime}\left(a^{\prime \prime}\right)^{-1}\right)^{\prime}=0, \quad u^{t} B^{\prime \prime}=\lambda a^{\prime \prime},
$$

and finally

$$
u^{t} B=\lambda a+\mu t+\nu
$$

which exhibits a $\mathbb{P}^{4}$ containing $X$, completing the proof of Proposition (3.5).

4. Degenerate families of matrices. Let $\{A(t)\}$ be a 1-parameter analytic family of $n \times n$ matrices. Fix an $s$ and put $B(t)=A(t+s)-$ $A(s)$. We will see that

$$
|B(t)|=\sigma_{0}(s) t^{n}+\sigma_{1}(s) t^{n+1}+\cdots .
$$

The conditions for $\{A(t)\}$ to be a degenerate family (3.0), $|B(t)| \equiv 0$, are

$$
\sigma_{i}(s)=0, i \geq 0 \text {. }
$$

The $1^{\text {st }}$ two coefficients are, as we'll soon see,

$$
\sigma_{0}=\left|A^{\prime}(s)\right| \quad \text { and } \quad \sigma_{1}=\frac{1}{2} \frac{d}{d s}\left|A^{\prime}(s)\right| \text {. }
$$

Of course, if $\sigma_{0} \equiv 0$ then also $\sigma_{1} \equiv 0$. We say that " $\sigma_{1} \equiv 0$ " imposes no inductive condition on the family $\{A(t)\}$.

Formulas for the other $\sigma$ 's are described by introducing the derivative operator, $D_{i}$, where $D_{i} B$ is the $n \times n$ matrix whose columns are the same as those of $B$, except for the $i$ th column, which is the derivative of the $i$ th column of $B$. A well-known formula states

$$
|B|^{\prime}=\sum\left|D_{i} B\right|, \quad i=1, \ldots, n .
$$

So

$$
|B|^{(N)}=\sum\left|D_{i_{1}} \cdots D_{i_{N}} B\right|=\sum\left(\begin{array}{c}
N \\
\alpha
\end{array}\right)\left|D_{1}^{\alpha_{1}} \cdots D_{n}^{\alpha_{n}} B\right|
$$


where the summation is over all $n$-tuples $\alpha=\left(\alpha_{1}, \ldots, \alpha_{n}\right)$ of nonnegative integers such that $|\alpha|=\alpha_{1}+\cdots+\alpha_{n}=N$. The binomial coefficient is

$$
\left(\begin{array}{c}
N \\
\alpha
\end{array}\right)=N !(\alpha !)^{-1} \quad \text { where } \alpha !=\alpha_{1} ! \cdots \alpha_{n} !
$$

The coefficients of the series (4.0) are found by evaluating (4.2) at $t=0$. But $B(0)=0$, so each term on the right side of (4.2) is zero unless each $\alpha_{j} \geq 1$; i.e. the series (4.0) begins at $t^{n}$.

We change notation and write $N=n+m, 1=(1,1, \ldots 1)$ and consider indices of the form $1+\alpha, \alpha_{j} \geq 0$. Since

$$
(n+m) ! \sigma_{m}=\left(\frac{d}{d t}\right)^{n+m}|B| \quad \text { at } t=0
$$

we conclude that

$$
\sigma_{m}=\sum \frac{1}{(1+\alpha) !}\left|D^{\alpha} A^{\prime}(s)\right|
$$

where the summation is over all $\alpha$ with $|\alpha|=m$, and $D^{\alpha}$ denotes $D_{1}^{\alpha_{1}} \ldots$ $D_{n}^{\alpha_{n}}$. We have used the relation $D_{1} \cdots D_{n} B=A^{\prime}(t+s)$.

The formulas (4.1.1) are now easy to verify. Using a modification of formula (4.3), I have written each of the conditions $\sigma_{2}=0, \sigma_{3}=0$ and $\sigma_{4}=0$ in terms of simple expressions involving $A^{\prime}(s)$. It turns out that " $\sigma_{3}=0$ " imposes no inductive condition on $\{A(t)\}$; it also appears that the odd relations $\sigma_{2 k+1}=0$ are implied by $\sigma_{0}=\cdots=\sigma_{2 k}=0$, but I have not verified this.

Let $\{E(t)\}$ denote a 1-parameter family of $n$-vectors. We denote the $n \times n$ Wronskian matrix by

$$
\omega(E)=\left(E E^{\prime} \cdots E^{(n-1)}\right)
$$

LEMMA (4.4). The Wronskian satisfies $|\omega(E)|=0$ precisely when there is a constant vector $u \neq 0$ with $u^{t} E \equiv 0$.

Proof. The existence of such a $u$ implies that $u^{t} \omega(E)=0$, hence $|\omega(E)|=0$. Conversely, assume that $|\omega(E)|=0$. For $n=1$, the result is trivial. So, assume that $n \geq 2$, and that the result is valid for matrices of size $n-1$. Over an open set in the parameter domain, we may construct an analytic family of $n$-vectors $\{u(t)\}$ such that

$$
u^{t} \omega(E)=0 \text {. }
$$

By rearranging the coefficients of $E$, we may assume that $u^{t}=\left(v^{t} 1\right)$, with $v$ an $n-1$ vector. If $v$ is constant, there is nothing more to prove, so 
assume that $v^{\prime} \neq 0$. Differentiating (4.4.1), we obtain

$$
u^{\prime t} \omega(E)+u^{t} \omega(E)^{\prime}=0 .
$$

Let $\tilde{E}$ denote the 1 st $n-1$ entries of $E$. From the 1 st $n-1$ columns of (4.4.2) we conclude that $v^{\prime t} \omega(\tilde{E})=0$. Thus $|\omega(\tilde{E})|=0$, and by the inductive assumption there is a constant $n-1$ vector $v$ with $v^{t} \tilde{E}=0$. Take $u^{t}=\left(v^{t} 0\right)$.

Recall (1.2) that the family $\{A(t)\}$ is called trivially degenerate if there is a constant $n$-vector $u \neq 0$ such that either $A^{\prime} u=0$ or $u^{t} A^{\prime}=0$.

We formulate, now,

Proposition (4.5). Let $\{A(t)\}, t \in U$, be a degenerate family of $n \times n$ matrices for which $A^{\prime}(t)$ has rank 1 for all $t \in U$. Then $\{A(t)\}$ is trivially degenerate. More precisely, express $A^{\prime}=E P^{t}$, where $E(t)$ and $P(t)$ are $n$-vectors defined over an open subset of $U$. We will see that either (i) $|\omega(E)|=0$, or (ii) $|\omega(P)|=0$, so that by Lemma (4.4) there is a constant $n$-vector $u \neq 0$ such that in case (i), $u^{t} A^{\prime}=0$, and in case (ii), $A^{\prime} u=0$.

(4.6) For an $n$-tuple of non-negative integers $\lambda=\left(\lambda_{1}, \ldots, \lambda_{n}\right)$, let $E^{(\lambda)}$ denote the product $E_{1}^{\left(\lambda_{1}\right)} \cdots E_{n}^{\left(\lambda_{n}\right)}$, and as usual $E^{(i)}=(d / d t)^{i} E$. Let $\left|E^{(\lambda)}\right|$ denote the determinant of the matrix $\left(E^{\left(\lambda_{1}\right)}, \ldots, E^{\left(\lambda_{n}\right)}\right)$.

Claim. The coefficients (4.0) may be expressed

$$
\sigma_{m}=\sum\left|E^{(\lambda)}\right|\left|P^{(\mu)}\right|(\lambda ! \mu !)^{-1} \delta(\lambda, \mu)
$$

where the summation is over all $\lambda, \mu \in \mathbb{N}^{n}$ with $0 \leq \lambda_{1}<\cdots<\lambda_{n}$, $0 \leq \mu_{1}<\cdots<\mu_{n},|\lambda|+|\mu|=m$. The coefficient $\delta(\lambda, \mu)$ is the determinant of the matrix $\left(1+\lambda_{i}+\mu_{j}\right)^{-1}, i, j=1, \ldots, n$, and is essentially the Cauchy double alternant $[3$, p. 311] for which the formula is

$$
\delta(\lambda, \mu)=\prod_{i>j}\left(\lambda_{i}-\lambda_{j}\right)\left(\mu_{i}-\mu_{j}\right) \prod_{i, j}\left(1+\lambda_{i}+\mu_{j}\right)^{-1}
$$

see [4, p. 348]. In particular, the $\delta(\lambda, \mu)$ that appear in (4.7) are not zero.

Assume for the moment that the claim is true. Let $M=n(n-1)$. The $\lambda$ 's and $\mu$ 's are strictly increasing, so $|\lambda|$ and $|\mu|$ are each greater than $0+1+\cdots+n-1=M / 2$. Thus,

$$
\sigma_{m}=0 \text { for } m<M,
$$


while

$$
\sigma_{M}=|\omega(E)||\omega(P)|(0 ! 1 ! \cdots(n-1) !)^{-2} \delta_{n} .
$$

Thus, " $\sigma_{M}=0$ " implies that $|\omega(E)||\omega(P)|=0$, and this proves the Proposition.

To prove the Claim, we substitute $A^{\prime}=E P^{t}$ into formula (4.3). The $i^{\text {th }}$ column of $D^{\alpha} A^{\prime}$ is

$$
\left(E P_{i}\right)^{\left(\alpha_{i}\right)}=\sum\left(\begin{array}{c}
\alpha_{i} \\
\lambda_{i}
\end{array}\right) E^{\left(\lambda_{t}\right)} P_{i}^{\left(\alpha_{t}-\lambda_{t}\right)}
$$

where the summation is over all $\lambda_{i} \geq 0$, with the convention that $P_{i}^{(r)}=0$ when $r<0$. Recalling notation (4.6), we may write

$$
\left|D^{\alpha} A^{\prime}\right|=\sum\left(\begin{array}{c}
\alpha \\
\lambda
\end{array}\right) P^{(\alpha-\lambda)}\left|E^{(\lambda)}\right|
$$

where

$$
\left(\begin{array}{l}
\alpha \\
\lambda
\end{array}\right)=\left(\begin{array}{c}
\alpha_{1} \\
\lambda_{1}
\end{array}\right) \cdots\left(\begin{array}{c}
\alpha_{n} \\
\lambda_{n}
\end{array}\right)
$$

and the sum is over all $\lambda_{i} \geq 0$. Substitution into (4.3) yields

$$
\begin{aligned}
\sigma_{m}=\sum_{\lambda}\left|E^{(\lambda)}\right|(\lambda !)^{-1} \sum_{\alpha}(1+\alpha)^{-1}(\alpha-\lambda) !^{-1} P^{(\alpha-\lambda)} \\
\quad \text { where }(1+\alpha)^{-1}=\left(\left(1+\alpha_{1}\right) \cdots\left(1+\alpha_{n}\right)\right)^{-1} .
\end{aligned}
$$

Next, arrange the $\lambda$ 's into increasing order. For $\sigma$ a permutation of $\{1, \ldots, n\}$, let $\left(\lambda_{\sigma}\right)_{i}=\lambda_{\sigma(i)}$; and $\operatorname{sg}(\sigma)= \pm 1$ is the sign of $\sigma$. Then (4.8) becomes

$$
\sigma_{m}=\sum_{\lambda}\left|E^{(\lambda)}\right|(\lambda !)^{-1} \sum_{\sigma} \operatorname{sg}(\sigma) \sum_{\alpha}(1+\alpha)^{-1}\left(\alpha-\lambda_{\sigma}\right) !^{-1} P^{\left(\alpha-\lambda_{\sigma}\right)}
$$

where, now, $0 \leq \lambda_{1} \leq \lambda_{2}<\cdots<\lambda_{n}$ and $\sigma$ runs through the permutations of $\{(1, \ldots, n)\}$. Let $\mu=\alpha-\lambda_{\sigma}$. Then

$$
\begin{aligned}
\sigma_{m} & =\sum_{\lambda}\left|E^{(\lambda)}\right|(\lambda !)^{-1} \sum_{\mu} P^{(\mu)}(\mu !)^{-1} \sum_{\sigma} \operatorname{sg}(\sigma)\left(1+\mu+\lambda_{\sigma}\right)^{-1} \\
& =\sum_{\lambda}\left|E^{(\lambda)}\right|(\lambda !)^{-1} \sum_{\mu} P^{(\mu)}(\mu !)^{-1} \delta(\lambda, \mu)
\end{aligned}
$$

and, upon rearranging the $\mu$ 's into increasing sequences, we arrive at formula (4.7). 


\section{REFERENCES}

[1] P. Griffiths and J. Harris, Algebraic Geometry and Local Differential Geometry, Ann. Scient. Éc. Norm. Sup. $4^{\mathrm{e}}$ série, t. 12 (1979), 355-432.

[2] C. M. Jessop, The Line Complex, Cambridge University Press 1903.

[3] T. Muir, The Theory of Determinants, Vol. III. Macmillan and Co. Ltd., London 1920.

[4] T. Muir and W. Metzler, The Theory of Determinants, Privately published, Albany, NY 1930.

Received December 1, 1983. This work was supported by a Natural Sciences and Engineering Research Council of Canada fellowship and grant.

UNIVERSITY OF BRITISH COLUMBIA

VANCOUVER, B.C. CANADA

V6T 1 Y4 


\section{PACIFIC JOURNAL OF MATHEMATICS EDITORS}

\author{
V. S. VARADARAJAN (Managing Editor) \\ University of California \\ Los Angeles, CA 90024 \\ Charles R. DePrima \\ California Institute of Technology \\ Pasadena, CA 91125 \\ R. FINN \\ Stanford University \\ Stanford, CA 94305
}

\author{
HeRmanN FlaschKa \\ University of Arizona \\ Tucson, AZ 85721 \\ Ramesh A. Gangolli \\ University of Washington \\ Seattle, WA 98195 \\ ROBION KIRBY \\ University of California \\ Berkeley, CA 94720
}

C. C. MOORE

University of California

Berkeley, CA 94720

H. SAMELSON

Stanford University

Stanford, CA 94305

HAROLD STARK

University of California, San Diego

La Jolla, CA 92093

ASSOCIATE EDITORS
R. ARENS
E. F. BECKENBACH
B. H. NeUmanN
F. WOLF
K. YosHIDA (1906-1982)

\section{SUPPORTING INSTITUTIONS}

UNIVERSITY OF ARIZONA

UNIVERSITY OF BRITISH COLUMBIA

CALIFORNIA INSTITUTE OF TECHNOLOGY

UNIVERSITY OF CALIFORNIA

MONTANA STATE UNIVERSITY

UNIVERSITY OF NEVADA, RENO

NEW MEXICO STATE UNIVERSITY

OREGON STATE UNIVERSITY
UNIVERSITY OF OREGON

UNIVERSITY OF SOUTHERN CALIFORNIA

STANFORD UNIVERSITY

UNIVERSITY OF HAWAII

UNIVERSITY OF TOKYO

UNIVERSITY OF UTAH

WASHINGTON STATE UNIVERSITY

UNIVERSITY OF WASHINGTON 


\section{Pacific Journal of Mathematics}

Vol. 119, No. $1 \quad$ May, 1985

Maurice Chacron, Nonisotropic unitary spaces and modules with

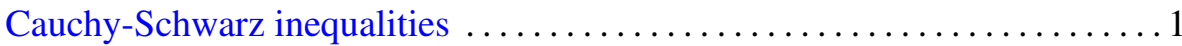

Myriam Dechamps-Gondim, Françoise Piquard and H. Queffélec, On

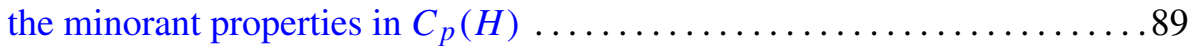

Klaus Floret and V. B. Moscatelli, On bases in strict inductive and projective limits of locally convex spaces .................... 103

Norman Joseph Goldstein, Degenerate secant varieties and a problem on

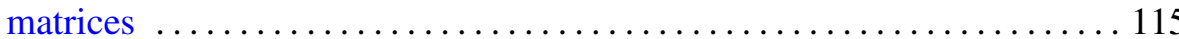

Harold Morris Hastings and Stefan Waner, $G$-bordism with singularities

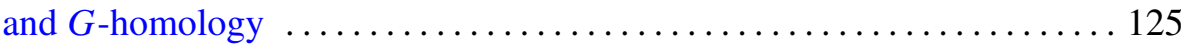

Takesi Isiwata, Clopen realcompactification of a mapping ........... 153

Hisao Kato, Concerning hyperspaces of certain Peano continua and strong

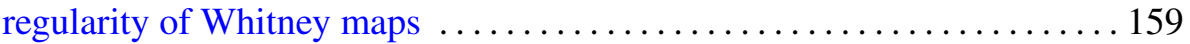

Elyahu Katz and Sidney Allen Morris, Free products of topological groups

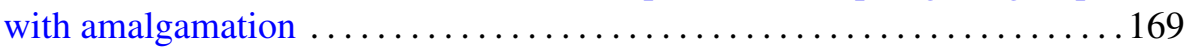

Kyewon Koh Park, Nice dense subsets for ergodic flows and Bernoulli

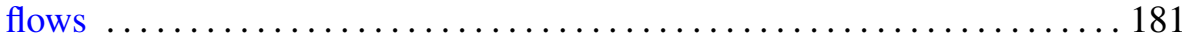

Francis Pastijn and Peter George Trotter, Lattices of completely regular

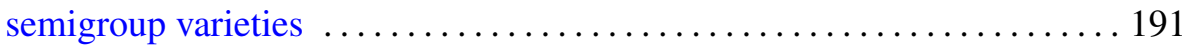

Rae Michael Andrew Shortt, Reticulated sets and the isomorphism of analytic powers

David A. Stegenga and Kenneth R. Stephenson, Generic covering properties for spaces of analytic functions

M. V. Subba Rao and R. Sitaramachandra Rao, On some infinite series of

L. J. Mordell and their analogues 